FACTA UNIVERSITATIS (NIŠ)

SER. MAth. Inform. Vol. 34, No 2 (2019), 261-273

https://doi.org/10.22190/FUMI1902261Y

\title{
BERTRAND-B CURVES IN THE THREE DIMENSIONAL SPHERE
}

\author{
Firat Yerlikaya and İsmail Aydemir
}

(C) 2019 by University of Niš, Serbia | Creative Commons Licence: CC BY-NC-ND

Abstract. We define a Bertrand-B curve $\alpha$ in the three dimensional sphere $\mathbb{S}^{3}(r)$ such that there exists an isometry $\phi$ of $\mathbb{S}^{3}(r)$, satisfying $(\phi \circ \beta)(s)=X(s, t(s))$ for another curve $\beta$ and both curves have common binormal geodesics at corresponding points. We analyze the condition of being Bertrand-B curves in $\mathbb{S}^{3}(r)$ and prove that the immersed curve with curvatures $\varepsilon_{1}, \varepsilon_{2}$ in $\mathbb{S}^{3}(r)$ is a Bertrand-B curve if and only if it satisfies $\varepsilon_{1}^{2}+\varepsilon_{2}^{2}=1$. Also, we analyze some conclusions about a pair of Bertrand-B curves in $\mathbb{S}^{3}(r)$. As an application, we give an example that the conclusions are verified.

Keywords. Bertrand-B curve; isometry; curvature.

\section{Introduction}

The theory of curves examines the geometric property of the plane and space curves by means of algebraic and calculus methods. The most common application areas of these methods are special curves such as helices, Bertrand curves, Mannheim curves, etc. The special curves in ambient spaces (semi-Euclidean space $\mathbb{R}_{v}^{n+1}$, Galilean space $G^{3}$, etc.) are generally characterized by the algebraic equations relating their curvature and torsion functions [1],[2],[3],[4]. For instance, Bertrand curves and Mannheim curves in the three dimensional Euclidean space $\mathbb{R}^{3}$ are characterized by, respectively;

$$
\lambda \kappa+\mu \tau=1 \text { and } \kappa=\lambda\left(\kappa^{2}+\tau^{2}\right)
$$

where $\lambda \neq 0$ and $\mu$ are some constants, $\kappa$ and $\tau$ are the curvature and torsion functions of these special curves, respectively [5],[6].

Naturally, it gives rise to the following question: Is it possible to extend the studies concerning the mentioned curves to 3-dimensional Riemannian or Lorentzian space forms? As an answer to this question, Choi et al. have given a definition

Received October 23, 2017; accepted January 31, 2019

2010 Mathematics Subject Classification. Primary 53B21; Secondary 53A40, 53A04 
of Bertrand curves in 3-dimensional Riemannian space forms $M$ and they have proved that a Frenet curve $\alpha$ with the curvature $\kappa$ and the torsion $\tau$ in $M$ is a Bertrand curve. It satisfies $\tau=0$ or $\kappa+a \tau=b$ for constants $a$ and $b \neq 0$ [7] as a necessary and sufficient condition. Recently, a definition of Mannheim curves in both Riemannian and Lorentzian space forms has also been given [8],[9]. All of the surveys mentioned above are done with reference to the Frenet-Serret frame which was adopted to formulate a space curve in ambient spaces. On the other hand, it is known that there are other frames in which all invariant properties of a space curve are investigated. These are called Bishop frames. The basic idea of creating such frames is to provide minimum bending. The minimum bending of space curves has a wide range of applications such as the creation of a continuous robot model from the analysis of the DNA structure [10], [11]. Thus, it is appropriate to expect all new types of curves that can be introduced on the Bishop frames to contribute to such areas of application.

The notion of Bertrand B-curves in 3-dimensional Euclidean space has been defined by Yerlikaya et al. and has been given the characterizations of Bertrand B-curves related to mate [12]. In this paper, we expand the definition of Bertrand B-curves to the three-dimensional sphere $\mathbb{S}^{3}(r)$ and give the algebraic qualification of Bertrand B-curves in $\mathbb{S}^{3}(r)$.

\section{Basic definitions and notations}

Let $\mathbb{S}^{3}(r)$ denote a three-dimensional sphere with the constant curvature $c=1$, defined by

$$
\mathbb{S}^{3}(r)=\left\{\left(x_{1}, x_{2}, x_{3}, x_{4}\right) \in \mathbb{R}^{4} \mid \sum_{i=1}^{4} x_{i}^{2}=r^{2}\right\}, \quad r>0 .
$$

Note that we regard $S^{3}(r)$ as a subcase of $\mathbb{R}^{4}$ equipped with the inner product for $x, y \in T_{p} S^{3}(r)$ :

$$
\langle x, y\rangle=x_{1} y_{1}+x_{2} y_{2}+x_{3} y_{3}+x_{4} y_{4}
$$

where $T_{p} S^{3}(r)$ denotes the tangent space of $S^{3}(r)$ at $p \in \mathbb{R}^{4}$. We also need to note the definition of wedge product (or cross product) in $\mathbb{R}^{4}$. If $x, y, z \in \mathbb{R}^{4}$, the vector $\langle x \times y \times z, w\rangle$ is defined as a unique one that satisfies $\langle x \times y \times z, w\rangle=$ $\operatorname{det}(x, y, z, w)$ for every $w \in \mathbb{R}^{4}$

Let $\alpha=\alpha(s): I \subset \mathbb{R} \rightarrow S^{3}(r)$ be an immersed curve and suppose, without loss of generality, that $\alpha$ is parametrized by the arc-length parameter and there exists an orthonormal frame $\{T, N, B\}$ with functions $\{\kappa, \tau\}$ (called the curvature and torsion of $\alpha$ ) along $\alpha$ (called the Frenet-Serret frame), satisfying the derivative formula

$$
\begin{aligned}
& \nabla_{T} T=\kappa N-\frac{1}{r^{2}} \alpha, \\
& \nabla_{T} N=-\kappa T+\tau B, \\
& \nabla_{T} B=-\tau N .
\end{aligned}
$$


where $\nabla$ symbolises the Levi-Civita connection of $\mathbb{R}^{4}$.

On the other hand, a new version of the Bishop frame in a three dimensional Euclidean space $\mathbb{R}^{3}$ is introduced by Yllmaz and Turgut such that type-2 Bishop and Frenet-Serret frames have a mutual vector field, i.e. binormal vector fields [13]. They present both a relationship between Frenet and Bishop vectors and type-2 Bishop derivative equation. From now on, it is possible to ask the following question in the light of the mentioned work.

Question: Is it possible to get the covariant derivative equations of type-2 Bishop for an immersed curve in $S^{3}(r)$ ?

As an answer to the above question, we need to be reminded of the following expressions:

Definition 2.1. The rotation matrix for two arbitrary vectors in the Euclidean plane is defined by the following expressions, respectively:

or

$$
\left(\begin{array}{cc}
\cos \theta(s) & -\sin \theta(s) \\
\sin \theta(s) & \cos \theta(s)
\end{array}\right)
$$

$$
\left(\begin{array}{cc}
\cos \theta(s) & \sin \theta(s) \\
-\sin \theta(s) & \cos \theta(s)
\end{array}\right)
$$

where $\theta(s)$ is the angle between two vectors [14].

Definition 2.2. Let $\alpha$ be an immersed curve in the three dimensional sphere $S^{3}(r)$. Then, the Gauss formula of $S^{3}(r)$ along $\alpha$ is given by the following equation for any vector field $X$ :

$$
X^{\prime}=\nabla_{s} X-\left\langle X, \alpha^{\prime}\right\rangle \alpha
$$

where' and $\nabla_{s}$ are symbolised by the natural differentiation of $\mathbb{R}^{4}$ and the covariant derivative of $S^{3}(r)$ along $\alpha$, respectively [14].

We can now express the covariant derivative equation of type-2 Bishop using the above definitions in $S^{3}(r)$ as follows:

$$
\begin{array}{cc}
\nabla_{s} \xi_{1}= & -\varepsilon_{1} B-\cos \theta(s) \alpha, \\
\nabla_{s} \xi_{2}= & -\varepsilon_{2} B+\sin \theta(s) \alpha, \\
\nabla_{s} B= & \varepsilon_{1} \xi_{1}+\varepsilon_{2} \xi_{2}
\end{array}
$$

Remark 2.1.

$$
\left[\begin{array}{l}
T \\
N \\
B
\end{array}\right]=\left[\begin{array}{ccc}
\cos \theta(s) & \sin \theta(s) & 0 \\
-\sin \theta(s) & \cos \theta(s) & 0 \\
0 & 0 & 1
\end{array}\right]\left[\begin{array}{l}
\xi_{1} \\
\xi_{2} \\
B
\end{array}\right]
$$

$$
\kappa=-\theta^{\prime}(s)
$$

$$
\cos \theta(s) \varepsilon_{1}(s)=-\sin \theta(s) \varepsilon_{2}(s) .
$$




\section{Bertrand-B curves in 3-dimensional sphere}

We begin by getting a crucial definition that is used to expand the concept of Bertrand-B curves to the sphere $S^{3}(r)$.

Definition 3.1. For $v \in T_{p} S^{3}(r)$, let $\gamma_{v}$ denote a unique maximal geodesic in $S^{3}(r)$ with the initial velocity $\gamma_{v}^{\prime}(0)=v$. Let

$$
U=\left\{v \in T S^{3}(r): 1 \in \text { domain } \gamma_{v}\right\}
$$

and let $\exp : U \rightarrow S^{3}(r)$ be defined by

$$
\exp _{p}(v)=\gamma_{v}(1)
$$

exp is called the exponential map of $S^{3}(r)$,where $U$ is an open set in $T S^{3}(r)$ and $p$ is the start point of $\gamma_{v}[14]$.

We can now give the definition of Bertrand-B curves:

Definition 3.2. Let $\alpha(s)$ be an immersed curve in a 3-dimensional simply connected space form $S^{3}(r)$ and $\left\{\xi_{1_{\alpha}}, \xi_{2_{\alpha}}, B_{\alpha}\right\}$ be type-2 Bishop of $\alpha$. With the aim of exponential map, a ruled surface $X_{B_{\alpha}}$ is defined such that

$$
X_{B_{\alpha}}(s, t)=\exp _{\alpha(s)}\left(t B_{\alpha}(s)\right) .
$$

An immersed curve $\beta=\beta(s)$ in $S^{3}(r)$ is said to be a Bertrand-B mate of $\alpha$ if the binormal vector field of $\beta$ determined by $\beta(s)=X_{B_{\alpha}}(s, t(s))$ is congruent to $B_{\alpha}\left(s_{0}\right)$ or $-B_{\alpha}\left(s_{0}\right)$ for each $s_{0}$. By the time an immersed curve $\alpha$ in $S^{3}(r)$ accepts its Bertrand-B mate, we call $\alpha$ a Bertrand-B curve in $S^{3}(r)$.

Another concept related to this exponential map: parallelism can be used to transport tangent vectors from one point of a surface to another. Accordingly, for $p \in S^{3}(r)$ and $v \in T_{p} S^{3}(r)$ with $\|v\|=1$ are considered as vectors in $\mathbb{R}^{4}$, a relationship between the exponential map and the parallel transport $P^{t}(v)$ as follows:

$$
\exp _{p}(t v)=\cos t p+\sin t v
$$

and

$$
P^{t}(v)=-\sin t p+\cos t v,
$$

In the light of the concepts described above, our goal is to find the condition of being a Bertrand-B curve for an arbitrary immersed curve in the three dimensional sphere:

For $\alpha=\alpha(s)$ let there be an immersed curve parametrized by arc-lenght in $S^{3}(r)$, let $\beta=\beta(\bar{s})$ with $\left\|\beta^{\prime}(\bar{s})\right\|=1$ be a Bertrand-B mate of $\alpha$. Note that we 
can assume, without loss of generality, that $\frac{d \bar{s}}{d s}>0$ and the curve $\beta(\bar{s})$ and $B_{\beta}(\bar{s})$, called its binormal vector field, stated by

$$
\begin{aligned}
\beta(\bar{s}) & =\exp _{\alpha(s)}\left(t(s) B_{\alpha}(s)\right) \\
& =\cos (t(s)) \alpha(s)+\sin (t(s)) B_{\alpha}(s)
\end{aligned}
$$

and $B_{\beta}(\bar{s})=P^{t(s)}\left(B_{\alpha}(s)\right)$, where $\beta(\bar{s})$ is the point in $\beta$ corresponding to $\alpha(s)$.

By taking the derivative of the equation 3.1 in $\mathbb{R}^{4}$ and applying the Gauss formula and the Bishop type-2 equation of $\alpha$, we get

$$
\begin{gathered}
\beta^{\prime}(\bar{s})=\{\cos (t(s))\}^{\prime} \alpha(s)+\frac{d s}{d \bar{s}}\left\{\cos (t(s)) \cos \theta(s)+\varepsilon_{1_{\alpha}}(s) \sin (t(s))\right\} \xi_{1_{\alpha}}(s) \\
+\frac{d s}{d \bar{s}}\left\{\cos (t(s)) \sin \theta(s)+\varepsilon_{2_{\alpha}}(s) \sin (t(s))\right\} \xi_{2_{\alpha}}(s)+\{\sin (t(s))\}^{\prime} B_{\alpha}(s) .
\end{gathered}
$$

Considering the fact that

$$
\left\langle\beta^{\prime}, B_{\beta}\right\rangle=0,\left\langle\beta^{\prime}, \beta\right\rangle=0
$$

and

$$
B_{\beta}=-\sin (t(s)) \alpha+\cos (t(s)) B_{\alpha}
$$

$\beta^{\prime}$ is orthogonal to $\alpha$ and $B_{\alpha}$ in $\mathbb{R}^{4}$. Thus from 3.2 , we easily get

$$
\{\cos (t(s))\}^{\prime}=\{\sin (t(s))\}^{\prime}=0 .
$$

Now that $t$ is a non-zero smooth function, $t(\bar{s})$ designate for $\mu \neq 0$. Besides, 3.1 and 3.2 are respectively determined by

$$
\beta(\bar{s})=\cos \mu \alpha(s)+\sin \mu B_{\alpha}(s)
$$

and

$$
\beta^{\prime}(\bar{s})=\frac{d s}{d \bar{s}}\left\{\begin{array}{c}
\cos \mu \cos \theta(s) \\
+\varepsilon_{1_{\alpha}}(s) \sin \mu
\end{array}\right\} \xi_{1_{\alpha}}(s)+\frac{d s}{d \bar{s}}\left\{\begin{array}{c}
\sin \mu \varepsilon_{2_{\alpha}}(s) \\
+\cos \mu \sin \theta(s)
\end{array}\right\} \xi_{2_{\alpha}}(\bar{s})
$$

from which,

$$
d \bar{s} / d s=\sqrt{\left(\cos \mu \cos \theta(s)+\varepsilon_{1_{\alpha}}(s) \sin \mu\right)^{2}+\left(\sin \mu \varepsilon_{2_{\alpha}}(s)+\cos \mu \sin \theta(s)\right)^{2}}
$$

or equivalently,

$$
d \bar{s} / d s=\sqrt{\cos ^{2} \mu+\sin ^{2} \mu\left(\varepsilon_{1_{\alpha}}^{2}(s)+\varepsilon_{2_{\alpha}}^{2}(s)\right)} .
$$


Now we can calculate the tangent vector of $\beta$ with regard to the Frenet vectors of $\alpha$ that is

$$
T_{\beta}(\bar{s})=c_{1}(s) T_{\alpha}(s)+c_{2}(s) N_{\alpha}(s)
$$

or bearing in mind that the equation 2.2

$T_{\beta}(\bar{s})=\left\{c_{1}(s) \cos \theta(s)-c_{2}(s) \sin \theta(s)\right\} \xi_{1_{\alpha}}(s)+\left\{c_{1}(s) \sin \theta(s)+c_{2}(s) \cos \theta(s)\right\} \xi_{2_{\alpha}}(s)$.

Equating the coefficients of the equations 3.5 and 3.8 , we get a linear equation system as follows:

$$
\begin{aligned}
& \cos \theta(s) c_{1}(s)-\sin \theta(s) c_{2}(s)=\frac{d s}{d \bar{s}}\left(\cos \mu \cos \theta(s)+\varepsilon_{1_{\alpha}}(s) \sin \mu\right) \\
& \sin \theta(s) c_{1}(s)+\cos \theta(s) c_{2}(s)=\frac{d s}{d \bar{s}}\left(\sin \mu \varepsilon_{2_{\alpha}}(s)+\cos \mu \sin \theta(s)\right) .
\end{aligned}
$$

Solving this system according to the cramer method, the functions $c_{1}$ and $c_{2}$ are determined such that

$$
\begin{gathered}
c_{1}(s)=\frac{d s}{d \bar{s}} \cos \mu \\
c_{2}(s)=\frac{d s}{d \bar{s}} \sin \mu\left(\cos \theta(s) \varepsilon_{2_{\alpha}}(s)-\sin \theta(s) \varepsilon_{1_{\alpha}}(s)\right)
\end{gathered}
$$

By taking the covariant derivative of 3.7 with regard to $\bar{s}$ in $\mathbb{R}^{4}$ and using the chain rule, the Gauss formula and the Frenet-Serret equation of $\alpha$, we get

$$
\begin{aligned}
\nabla_{\bar{s}} T_{\beta}(\bar{s}) & =\left\{\cos \mu-c_{1}(s) \frac{d s}{d \bar{s}}\right\} \alpha(s)+\left\{c_{1}{ }^{\prime}(s)-c_{2}(s) \kappa_{\alpha}(s) \frac{d s}{d \bar{s}}\right\} T_{\alpha}(s) \\
& +\left\{c_{2}{ }^{\prime}(s)+c_{1}(s) \kappa_{\alpha}(s) \frac{d s}{d \bar{s}}\right\} N_{\alpha}(s)+\left\{\sin \mu+c_{2}(s) \sqrt{\varepsilon_{1_{\alpha}}^{2}+\varepsilon_{2_{\alpha}}^{2}} \frac{d s}{d \bar{s}}\right\} B_{\alpha}(s)
\end{aligned}
$$

In what follows, since $\nabla_{\bar{s}} T_{\beta}(\bar{s})$ is proportional to

$$
N_{\beta}(\bar{s})=c_{3}(s) T_{\alpha}(s)+c_{4}(s) N_{\alpha}(s),
$$

it reduces to

$$
\nabla_{\bar{s}} T_{\beta}(\bar{s})=\left\{c_{1}{ }^{\prime}(s)-c_{2}(s) \kappa_{\alpha}(s) \frac{d s}{d \bar{s}}\right\} T_{\alpha}(s)+\left\{c_{2}{ }^{\prime}(s)+c_{1}(s) \kappa_{\alpha}(s) \frac{d s}{d \bar{s}}\right\} N_{\alpha}(s) .
$$

Lemma 3.1. Let $\alpha(s)$ be an immersed curve parametrized by arc-length and let $\beta(\bar{s})$ be a Bertrand- $B$ mate with $\left\|\beta^{\prime}(\bar{s})\right\|=1$ in the three dimensional sphere $S^{3}(r)$. Then, the following equalities hold: 
1. $\cos \mu-c_{1}(s) \frac{d s}{d \bar{s}}=0$

2. $\sin \mu+c_{2}(s) \sqrt{\varepsilon_{1_{\alpha}}^{2}+\varepsilon_{2_{\alpha}}^{2}} \frac{d s}{d \bar{s}}=0$.

We can now evaluate two cases from the lemma 3.1 as follows:

Case 1.From lemma 3.1, we have

$$
\cos \mu-c_{1}(s) \frac{d s}{d \bar{s}}=0 .
$$

Then, from the equations 3.6 and 3.9 and the necessary arrangement, we can write

$$
\frac{\sin ^{2} \mu \cos \mu\left(\left(\varepsilon_{1_{\alpha}}^{2}+\varepsilon_{2_{\alpha}}^{2}\right)-1\right)}{\cos ^{2} \mu+\sin ^{2} \mu\left(\varepsilon_{1_{\alpha}}^{2}+\varepsilon_{2_{\alpha}}^{2}\right)}=0 .
$$

Subcase 1.1. Let $\cos \mu=0.3 .6$ and 3.9- 3.10 is reduced to $c_{1}=0, c_{2}= \pm 1$ and $d \bar{s} / d s=\sqrt{\varepsilon_{1_{\alpha}}^{2}+\varepsilon_{2_{\alpha}}^{2}}$. According to the previous expressions, from 3.7, we get $T_{\beta}= \pm N_{\alpha}$. Then, apply these to 3.11 :

$$
\nabla_{\bar{s}} T_{\beta}(\bar{s})= \pm \frac{\kappa_{\alpha}}{\sqrt{\varepsilon_{1_{\alpha}}^{2}+\varepsilon_{2_{\alpha}}^{2}}} T_{\alpha}(s) .
$$

We distinguish four subcases according to the sign of the vector field.

Subsubcase 1.1.S1. $\left(T_{\beta}=N_{\alpha}, \nabla_{\bar{s}} T_{\beta}(\bar{s})>0\right)$. Eq. 3.12 becomes

$$
\nabla_{\bar{s}} T_{\beta}(\bar{s})=\frac{\kappa_{\alpha}}{\sqrt{\varepsilon_{1_{\alpha}}^{2}+\varepsilon_{2_{\alpha}}^{2}}} T_{\alpha}(s)
$$

from which, $\left\|\nabla_{\bar{s}} T_{\beta}(\bar{s})\right\|=\frac{\kappa_{\alpha}}{\sqrt{\varepsilon_{1_{\alpha}}^{2}+\varepsilon_{2_{\alpha}}^{2}}}$ and $N_{\beta}=T_{\alpha}$. From the wedge product in $E^{4}, B_{\beta}$ is given by

$$
B_{\beta}=-\sin \mu \alpha+\cos \mu B_{\alpha}=P^{\mu}\left(B_{\alpha}\right) .
$$

Thus $\beta$ is a Bertrand-B mate of $\alpha$.

Reasoning as in the subsubcase 1.1 , one says whether $\beta$ is a Bertrand-B mate of $\alpha$ or not.

Subcase 1.2. Let $\sin \mu=0$, say $\mu=\pi k, k \in \mathbb{Z}$. Thus, $\beta$ is isometric to $\alpha$.

Subcase 1.3. Let $\varepsilon_{1_{\alpha}}^{2}+\varepsilon_{2_{\alpha}}^{2}=1$. Eqs. (3.6) and (3.9)-(3.10) is reduced to $d s=d \bar{s}$, $c_{1}=\cos \mu$ and $c_{2}= \pm \sin \mu$. According to the previous expressions, from 3.7, we get $T_{\beta}(\bar{s})=\cos \mu T_{\alpha}(s) \pm \sin \mu N_{\alpha}(s)$. Then, apply these to 3.11 :

$$
\nabla_{\bar{s}} T_{\beta}(\bar{s})=\kappa_{\alpha}(s)\left\{ \pm \sin \mu T_{\alpha}(s)+\cos \mu N_{\alpha}(s)\right\}
$$


from which,

$$
\left\|\nabla_{\bar{s}} T_{\beta}(\bar{s})\right\|=\left|\kappa_{\alpha}(s)\right|
$$

Note that if $\kappa_{\alpha}(s)=0$, then $\nabla_{\bar{s}} T_{\beta}(\bar{s})=0$, that is, $\beta$ is a geodesic in $S^{3}$. Its principal normal vector field $N_{\beta}$ is given by

$$
N_{\beta}(\bar{s})= \pm \sin \mu T_{\alpha}(s)+\cos \mu N_{\alpha}(s)
$$

Considering the wedge product in $E^{4}$, the binormal vector field $B_{\beta}$ is obtained by

$$
\begin{gathered}
B_{\beta}(\bar{s})=\sin \mu \alpha(s)-\cos \mu B_{\alpha}(s) \\
=P^{\mu}\left(B_{\alpha}((s))\right) .
\end{gathered}
$$

Thus $\beta$ is a Bertrand-B mate of $\alpha$.

Case 2. From the lemma 3.1, we have

$$
\sin \mu+c_{2}(s) \sqrt{\varepsilon_{1_{\alpha}}^{2}+\varepsilon_{2_{\alpha}}^{2}} \frac{d s}{d \bar{s}}=0
$$

Then, from the equations 3.6 and 3.10 and the necessary arrangement, we can write

$$
\frac{\sin \mu \cos ^{2} \mu\left(1-\left(\varepsilon_{1_{\alpha}}^{2}+\varepsilon_{2_{\alpha}}^{2}\right)\right)}{\cos ^{2} \mu+\sin ^{2} \mu\left(\varepsilon_{1_{\alpha}}^{2}+\varepsilon_{2_{\alpha}}^{2}\right)}=0 .
$$

In this case, it is clear that the curve $\beta$ is again a Bertrand-B mate of $\alpha$, examined as in the case 1 .

Proposition 3.1. Let $\alpha=\alpha(s)$ be an immersed curve parametrized by arc-lenght in $S^{3}(r)$ with curvatures $\varepsilon_{1_{\alpha}}$ and $\varepsilon_{2_{\alpha}}$ and $\beta(\bar{s})=\cos \mu \alpha(s)+\sin \mu B_{\alpha}(s)$. Then, we have

- When $0<\mu<\frac{\pi}{2}, \beta$ is not a Bertrand-B mate of $\alpha$.

- When $\frac{\pi}{2}<\mu<\pi, \beta$ is a Bertrand-B mate of $\alpha$

- If $\kappa_{\alpha}=0$ then $\beta$ is a geodesic in $S^{3}(r)$.

Theorem 3.1. Let $\alpha=\alpha(s)$ be an immersed curve in the 3-dimensional sphere $S^{3}(r)$ with curvatures $\varepsilon_{1_{\alpha}}$ and $\varepsilon_{2_{\alpha}}$. Then, $\alpha$ is a Bertrand-B curve if and only if $\varepsilon_{1_{\alpha}}^{2}+\varepsilon_{2_{\alpha}}^{2}=1$.

After finding the condition of being a Bertrand-B curve in the three dimensional sphere $S^{3}(r)$, we can now give results concerning a pair of Bertrand-B curves: 
Let $\alpha(s)$ and $\beta(\bar{s})$ be a pair of Bertrand-B curves having the Bishop type-2 frames $\left\{\xi_{1_{\alpha}}, \xi_{2_{\alpha}}, B_{\alpha}\right\}$ and $\left\{\xi_{1_{\beta}}, \xi_{2_{\beta}}, B_{\beta}\right\}$, respectively, then there exists a differentiable function $t(\bar{s})$ such that

$$
\beta(\bar{s})=\cos (t(s)) \alpha(s)+\sin (t(s)) B_{\alpha}(s)
$$

where $\beta(\bar{s})$ is the point in $\beta$ corresponding to $\alpha(s)$.

Proposition 3.2. Let $\alpha$ and $\beta$ be a pair of Bertrand-B curves in $\mathbb{S}^{3}$. Then the following properties hold:

1. The function $t(\bar{s})$ is constant.

2. The angle between the tangent vectors $T_{\alpha}(s)$ and $T_{\beta}(\bar{s})$ at corresponding points equals to $\mu$.

3. The angle between $\xi_{1_{\alpha}}$ and $\xi_{1_{\beta}}$ vectors at corresponding points is constant.

4. The angle between $\xi_{2_{\alpha}}$ and $\xi_{2_{\beta}}$ vectors at corresponding points is constant.

Proof. (1) It can be seen that the function $t(\bar{s})$ is the constant from Eq. (3.4), which completes the proof.

(2) Taking the derivative of Eq. (3.1) with respect to $s$ in $\mathbb{R}^{4}$, we have

$$
T_{\beta}(\bar{s}) \frac{d \bar{s}}{d s}=\cos \mu T_{\alpha}(s)+\sin \mu\left\{\xi_{1_{\alpha}}(s) \varepsilon_{1_{\alpha}}(s)+\xi_{2_{\alpha}}(s) \varepsilon_{2_{\alpha}}(s)\right\} .
$$

By multiplying the previous equation with $T \alpha(s)$, we get

$$
\left\langle T_{\beta}(\bar{s}), T_{\alpha}(s)\right\rangle=\cos \mu+\sin \mu\left\{\varepsilon_{1_{\alpha}}(s) \cos \theta(s)+\varepsilon_{2_{\alpha}}(s) \sin \theta(s)\right\}
$$

where we use $\left\langle\xi_{1_{\alpha}}(s), T_{\alpha}(s)\right\rangle=\cos \theta(s)$ and $\left\langle\xi_{2_{\alpha}}(s), T_{\alpha}(s)\right\rangle=\sin \theta(s)$. Finally, taking into account Eq.(2.4), we deduce (2).

(3) By a straightforward computation, we get

$$
\begin{aligned}
\frac{d}{d s}\left\langle\xi_{1_{\alpha}}(s), \xi_{1_{\beta}}(\bar{s})\right\rangle= & -\varepsilon_{1_{\alpha}}(s)\left\langle B_{\alpha}(s), \xi_{1_{\beta}}(\bar{s})\right\rangle-\cos \theta(s)\left\langle\alpha(s), \xi_{1_{\beta}}(\bar{s})\right\rangle \\
& -\frac{d \bar{s}}{d s} \varepsilon_{1_{\beta}}(\bar{s})\left\langle\xi_{1_{\alpha}}(s), B_{\beta}(\bar{s})\right\rangle-\frac{d \bar{s}}{d s} \cos \theta(s)\left\langle\xi_{1_{\alpha}}(s), \beta(\bar{s})\right\rangle,
\end{aligned}
$$

that jointly with (3.1), (3.3) and $\xi_{1_{\beta}} \in S p\left\{T_{\alpha}, N_{\alpha}\right\}$ yields

$$
\frac{d}{d s}\left\langle\xi_{1_{\alpha}}(s), \xi_{1_{\beta}}(\bar{s})\right\rangle=0,
$$

which completes the claim.

(4) Similarly as in the item (b), one can see that the proof of the claim can be ended. 
Theorem 3.2. Let $\alpha$ and $\beta$ be a pair of Bertrand- $B$ curves in $\mathbb{S}^{3}$ and let $\kappa_{\alpha}$ and $\kappa_{\beta}$ be the curvatures of the pair, respectively. Then there exists a constant $\mu$ and $\eta$ such that the following relations hold:

$$
\begin{aligned}
& \text { 1. } \kappa_{\beta}^{2}(\bar{s})=\cos ^{2} \mu \kappa_{\alpha}^{2}(s)+\sin ^{2} \mu\left\{\varepsilon_{1_{\alpha}}^{\prime^{2}}(s)+\varepsilon_{2_{\alpha}}^{\prime^{2}}(s)\right\} \\
& \text { 2. } \kappa_{\alpha}^{2}(s)=\cos ^{2} \mu \kappa_{\beta}^{2}(\bar{s})+\sin ^{2} \mu\left\{\varepsilon_{1_{\beta}}^{\prime^{2}}(\bar{s})+\varepsilon_{2_{\beta}}^{2^{2}}(\bar{s})\right\} \\
& \text { 3. } \cos 2 \eta=\cos 2 \mu\left\{\varepsilon_{1_{\alpha}}(s) \varepsilon_{1_{\beta}}(\bar{s})+\varepsilon_{2_{\alpha}}(s) \varepsilon_{2_{\beta}}(\bar{s})\right\}
\end{aligned}
$$

where $\varepsilon_{1_{\alpha}}, \varepsilon_{2_{\alpha}}, \varepsilon_{1_{\beta}}$ and $\varepsilon_{2_{\beta}}$ stand for the curvatures of $\alpha$ and $\beta$, respectively.

Proof. (1) By the covariant derivative of Eq. (3.1), we get Eq. (3.5). Using Eq. (2.2) for the curve $\beta$, we have the following equation

$$
\begin{aligned}
\left\{\cos \theta(\bar{s}) \xi_{1_{\beta}}(\bar{s})+\sin \theta(\bar{s}) \xi_{2_{\beta}}(\bar{s})\right\} \frac{d \bar{s}}{d s}= & \left\{\cos \mu \cos \theta(s)+\sin \mu \varepsilon_{1_{\alpha}}(s)\right\} \xi_{1_{\alpha}}(s) \\
& +\left\{\cos \mu \sin \theta(s)+\sin \mu \varepsilon_{2_{\alpha}}(s)\right\} \xi_{2_{\alpha}}(s) .
\end{aligned}
$$

On the other hand, we have a constant angle $\eta$ because of the items (3) and (4) of Proposition (3.2), thus we can write

$$
\begin{aligned}
& \cos [\theta(\bar{s})-\eta]=\frac{d s}{d \bar{s}}\left\{\cos \mu \cos \theta(s)+\sin \mu \varepsilon_{1_{\alpha}}(s)\right\} \\
& \sin [\theta(\bar{s})-\eta]=\frac{d s}{d \bar{s}}\left\{\cos \mu \sin \theta(s)+\sin \mu \varepsilon_{2_{\alpha}}(s)\right\} .
\end{aligned}
$$

By taking the derivative of Eqs. (3.15) and (3.16) wrt $s$ in $\mathbb{R}^{4}$ and applying Eqs. (2.3) and (2.4), we deduce (1). This ends the proof.

(2) Now we have to hold Eq. (3.1) according to the curve $\alpha$ :

$$
\alpha(s)=\cos \mu \beta(\bar{s})+\sin \mu B_{\beta}(\bar{s}) .
$$

Thus, a straightforward computation leads to the following two equations:

$$
\begin{aligned}
& \cos [\theta(s)+\eta]=\frac{d \bar{s}}{d s}\left\{\cos \mu \cos \theta(\bar{s})-\sin \mu \varepsilon_{1_{\beta}}(\bar{s})\right\} \\
& \sin [\theta(s)+\eta]=\frac{d \bar{s}}{d s}\left\{\cos \mu \sin \theta(\bar{s})+\sin \mu \varepsilon_{2_{\beta}}(\bar{s})\right\} .
\end{aligned}
$$

By following a similar path in the item (a), one can easily see that the claim concludes.

(3) It is a consequence of the way followed by the multiplication of the Eqs. (3.15-3.17) and (3.16-3.18). 
Example 3.1. Let $\alpha=\alpha(s)$ be a model helix in the 3-dimensional sphere $\mathbb{S}^{3}(r)$, given by

$$
\alpha(s)=(\cos \phi \cos (a s), \cos \phi \sin (a s), \sin \phi \cos (b s), \sin \phi \sin (b s)),
$$

where $s$ is arc-length when $a^{2} \cos ^{2} \phi+b^{2} \sin ^{2} \phi=1$. Also, a straightforward computation gives us the following Frenet apparatus of $\alpha$ :

$$
\left\{\begin{array}{c}
T_{\alpha}(s)=(-a \cos \phi \sin (a s), a \cos \phi \cos (a s),-b \sin \phi \sin (b s), b \sin \phi \cos (b s)) \\
N_{\alpha}(s)=(-\sin \phi \cos (a s),-\sin \phi \sin (a s), \cos \phi \cos (b s), \cos \phi \sin (b s)) \\
B_{\alpha}(s)=(-b \sin \phi \sin (a s), b \sin \phi \cos (a s), a \cos \phi \sin (b s),-a \cos \phi \cos (b s)) \\
\kappa_{\alpha}=\sqrt{\left(a^{2}-1\right)\left(1-b^{2}\right)} \\
\tau_{\alpha}=a b
\end{array}\right.
$$

By applying the first two equations of the apparatus to (2.2), we get the Bishop-type 2 vector field as follows:

$$
\begin{array}{r}
\xi_{1_{\alpha}}(s)=(-f(s) \sin (a s)+\sin \phi \sin \theta(s) \cos (a s), f(s) \cos (a s)+\sin \phi \sin \theta(s) \sin (a s), \\
-g(s) \sin (b s)-\cos \phi \sin \theta(s) \cos (b s), \\
g(s) \cos (b s)-\cos \phi \sin \theta(s) \sin (b s)),
\end{array}
$$

where $f(s)=a \cos \phi \cos \theta(s)$ and $g(s)=b \sin \phi \cos \theta(s)$. Similarly, we get the remaining vector field $\xi_{2_{\alpha}}(s)$. Note a case that if $b=\frac{1}{a}$, then the curve $\alpha$ is a helix in $\mathbb{S}^{3}(r)$ parametrized by arc-length with $\tau=1$. In such a case, by taking the covariant derivative $\xi_{1_{\alpha}}(s)$ and $\xi_{2_{\alpha}}(s)$ with respect to $s$ and using the derivative formula of the Bishop type 2 , we obtain the following Bishop type 2 curvatures:

$$
\varepsilon_{1_{\alpha}}(s)=-\sin \theta(s), \quad \varepsilon_{2_{\alpha}}(s)=-\cos \theta(s)
$$

from which

$$
\varepsilon_{1_{\alpha}}^{2}(s)+\varepsilon_{2_{\alpha}}^{2}(s)=1 .
$$

This means that the curve $\alpha$ with $\tau=1$ is a Bertrand-B curve in $\mathbb{S}^{3}(r)$. Morever, the Bertrand-B partner curve $\beta$ of $\alpha$ is given by $\beta(\bar{s})=\cos \mu \alpha(\bar{s})+\sin \mu B_{\alpha}(\bar{s})$ with

$\bar{s}=\sqrt{\cos ^{2} \mu+\sin ^{2} \mu\left\{\varepsilon_{1_{\alpha}}^{2}(s)+\varepsilon_{2_{\alpha}}^{2}(s)\right\}} s$. Observe that the last equation supports Eq. (3.6). Furthermore, by calculating the curvature of $\beta$, it is easy see that the item (1) of the theorem (3.2) is satisfied when the curve $\alpha$ is a Bertrand-B curve, i.e. for $a b=1$ :

$$
\kappa_{\beta}(s)=\sqrt{\left(a^{2}-1\right)\left(1-b^{2}\right)+\sin ^{2} \mu\left\{\left((a b)^{2}-1\right)\left(a^{2}+b^{2}-2\right)\right\}}
$$

Consequently, we have the following proposition:

Proposition 3.3. A model helix with $\tau=1$ in the 3-dimensional sphere $\mathbb{S}^{3}(r)$ is a Bertrand-B curve. Moreover, the Bertrand-B partner curve of a model helix in $\mathbb{S}^{3}(r)$ is also a Bertrand-B curve. 


\section{Conclusion}

In this paper, we obtained a lemma which states the condition of what it takes to be a Bertrand-B curve. In creating this lemma, we used another curve (mentioned as $\beta(\bar{s}))$ as its mate and saw that it is possible for these curves to be Bertrand-B curves only if their mates exist. In addition, some conclusions about a pair of Bertrand-B curves in the three dimensional sphere (called a special Riemannian manifold) are stated. On the other hand, recent studies show that Bishop frames have attracted the attention of many scientists and geometers due to various applications in areas from engineering to computer graphics. Hence, we hope that the results of this study will serve the areas of application associated with Bishop frames.

\section{RE F E R E N C ES}

1. M. Barros, General helices and a theorem of lancret, Proceedings of the American Mathematical Society 125 (5) (1997) 1503-1509.

2. K. Orbay, E. Kasap, On mannheim partner curves in e3, International Journal of Physical Sciences 4 (5) (2009) 261-264.

3. A. O. Öğrenmiş, H. Öztekin, M. Ergüt, Bertrand curves in galilean space and their characterizations, Kragujevac Journal of Mathematics 32 (32) (2009) 139-147.

4. M. Y. Yilmaz, M. Bektaş, General properties of bertrand curves in riemann-otsuki space, Nonlinear Analysis: Theory, Methods \& Applications 69 (10) (2008) 3225-3231.

5. J. Bertrand, Mémoire sur la théorie des courbes à double courbure., Journal de Mathématiques Pures et Appliquées (1850) 332-350.

6. R. Blum, A remarkable class of mannheim curves, Canad. Math. Bull 9 (2) (1966) $223-228$.

7. J. H. Choi, T. H. Kang, Y. H. Kim, Bertrand curves in 3-dimensional space forms, Applied Mathematics and Computation 219 (3) (2012) 1040-1046.

8. J. H. Choi, T. H. Kang, Y. H. Kim, Mannheim curves in 3-dimensional space forms, Bulletin of the Korean Mathematical Society 50 (4) (2013) 1099-1108.

9. W. Zhao, D. Pei, X. Cao, Mannheim curves in nonflat 3-dimensional space forms, Advances in Mathematical Physics 2015.

10. L. Qiang, B. He, Kinematics and energy minimization approach for continuum robot, In Advances in Reconfigurable Mechanisms and Robots II (2016) 817-827.

11. C. Gregory S., Framed curves and knotted dna, Biochemical Society Transactions 41 (2013) 635-638.

12. F. Yerlikaya, S. Karaahmetoglu, I. Aydemir, On the bertrand b-pair curves in 3dimensional euclidean space, Journal of Science and Arts (3) (2016) 215-224.

13. S. Yılmaz, M. Turgut, A new version of bishop frame and an application to spherical images, Journal of Mathematical Analysis and Applications 371 (2) (2010) 764-776.

14. J. A. Thorpe, Elementary topics in differential geometry, Springer Science \& Business Media, 2012. 
Firat Yerlikaya

Faculty of Science and Arts

Department of Mathematics

P. O. Box 55139

Samsun, Turkey

firat.yerlikaya@omu.edu.tr

İsmail Aydemir

Faculty of Science and Arts

Department of Mathematics

P.O. Box 55139

Samsun, Turkey

iaydemir@omu.edu.tr 